\title{
MOLECULAR ANALYSIS OF TRANSLOCATIONS ASSOCIATED WITH EWING'S SARCOMA IN THE DIFFERENTIAL DIAGNOSIS OF UNDIFFERENTIATED AND UNCLASSIFIED SARCOMAS
}

Leonardo R. Soares¹, Marise A. R. Moreira1, Paula O. C. Queiroz¹, Hugo A. Bayeh¹, Izabela C. S. Albuquerque1, Miguel A. C. Coutinho', Débora F. Rodrigues ${ }^{1}$, Ruffo Freitas-Junior ${ }^{1}$

${ }^{1}$ Brazilian Mastology Research Network - Goiânia (GO), Brazil.

Undifferentiated and unclassified sarcomas constitute a heterogeneous group of sarcomas, including the Ewing sarcoma family of tumors (ESFT). However, some tumors with Ewing sarcoma (ES) morphology remain with undifferentiated histogenesis due to negative or non-informative molecular results. A case of 25-year-old female patient with a massive lesion occupying almost all of the mammary parenchyma on the right, with a hardened appearance and measuring $18.0 \mathrm{~cm}$ was described. Chest tomography and resonance showed extension of the lesion to the chest wall, with no apparent bone involvement. A histology of the material from core biopsy revealed high grade fusocellular neoplasia with areas of necrosis, atypia and mitosis; and absence of epithelial component represented in the sample. An immunohistochemistry of the lesion revealed negative expression of smooth muscle actin, HHF35, desmin, S100, CD34 and EMA. Positive expression of CD99, CD56 and CD57 markers was also observed. The patient underwent radical surgery and adjuvant treatment with chemotherapy and radiotherapy. In view of the immunohistochemically profile and rosette arrangement in a fusocellular lesion, a molecular evaluation of translocations associated with ES was performed. However, there were no translocations commonly associated with this pathology $[\mathrm{t}(11 ; 22)$ (q24; q12)]. The present report may contribute to a diagnostic investigation of similar cases and to the pre-test orientation of molecular evaluation. 